\title{
Laser vaginal rejuvenation: not ready for prime time-response to comments by Maggiore et al.
}

\author{
Alex Digesu \\ Published online: 27 March 2015 \\ (C) The International Urogynecological Association 2015
}

Dear Editor,

I would like to thank Dr Maggiore and colleagues for their input and remarks on our original editorial "Laser vaginal rejuvenation: not ready for prime time" $[1,2]$, as well as congratulate them on their work and contribution to a growing body of evidence-based techniques for managing important aspects of sexual dysfunction and vulvovaginal atrophy. However, I feel that their remarks reflect a misinterpretation and thus they are missing the major point of our editorial.

While I am in complete agreement that the widespread clinical application of the $\mathrm{CO}_{2}$ laser as a surgical tool in some areas of modern medicine is both significant and beneficial, its other uses and advantages and disadvantages are beyond the scope of the original editorial. We did not suggest that the $\mathrm{CO}_{2}$ laser did not have any clinical or surgical indication, but merely that its application in the context of genital rejuvenation needs to be very cautiously appraised, as currently there is a lack of a robust, qualitative body of data on the therapeutic advantages of the $\mathrm{CO}_{2}$ laser. The claim by Maggiore et al. that laser vaginal rejuvenation is popular in that it improves certain aspects of appearance and sexual function, according to our editorial, has not yet been substantiated by a large enough body of evidence.

To this end, it must be reiterated that there is an imminent and important need to build on the small case studies, such as those reported by Maggiore et al., in the form of a larger, multi-centred study. While we accept and commend the efforts of Dr Maggiore and colleagues, who have published several recent articles, including the short-term 12-week follow-up and single-centre pilot studies on the efficacy of $\mathrm{CO}_{2}$ laser therapy for vulvovaginal atrophy and dyspareunia, to our knowledge, their results for these conditions have not been reproduced by any other authors to date. This is despite the fact that these treatments have been offered to women for over 10 years.

We continue to feel strongly that there still remains a significantly under-regulated and potentially dangerous practice of laser vaginal rejuvenation driven through potentially misleading marketing and a subsequent misguided uptick of this intervention, whether through laser $\mathrm{CO}_{2}$ therapy or otherwise. We feel that the therapeutic advantages of the $\mathrm{CO}_{2}$ laser in the context of vaginal rejuvenation can only be realistically upheld on the back of conclusive, reproducible, objective and wide-ranging, reliable trials.

\section{References}

1. Maggiore ULR, Candiani M, Salvatore S (2015) Comments on Singh et al.: laser vaginal rejuvenation: not ready for prime time. Int Urogynecol J. doi:10.1007/s00192-015-2644-6

2. Singh A, Swift S, Khullar V, Digesu GA (2014) Laser vaginal rejuvenation: not ready for prime time. Int Urogynecol J 26(2):163-164. doi: 10.1007/s00192-014-2588-2

A. Digesu $(\bowtie)$

Academic Department of Obstetrics and Gynaecology, Imperial College Healthcare NHS Trust, South Wharf Road, London W2 $1 \mathrm{NY}, \mathrm{UK}$

e-mail: a.digesu@imperial.ac.uk 